\title{
Evaluation of mist production and tissue dissection efficiency using different types of ultrasound shears
}

\author{
A. Schneider $\cdot$ E. Doundoulakis $\cdot$ S. Can · \\ A. Fiolka · D. Wilhelm $\cdot$ H. Feußner
}

Received: 27 October 2008/Accepted: 18 April 2009/Published online: 23 May 2009

(C) Springer Science+Business Media, LLC 2009

\begin{abstract}
Background Ultrasound shears often are applied in minimally invasive surgery because they facilitate fast and secure tissue dissection, thereby reducing operative time. Although the technical principle underlying all the shears is almost identical, considerable differences exist between specific instruments. However, production of disturbing mist should be avoided.

Methods To obtain quantitative measurements regarding mist production, a novel hermetically sealed test system was developed. Tissue dissection efficiency was evaluated by means of a standardized cutting test. The dissection time and the numbers of cuttings were recorded. In this study, four different ultrasound dissectors from three manufacturers were assessed. One manufacturer provided two instruments: a conventional instrument and an improved version, which was designed particularly to reduce mist emission.

Results The fastest ultrasound dissector emitted the highest quantity of disturbing mist. However, improved dissection efficiency does not linearly correlate with mist production. This clearly could be shown for the improved "less mist production instrument," which turned out to
\end{abstract}

A. Schneider · E. Doundoulakis · S. Can · A. Fiolka ·

D. Wilhelm · H. Feußner

Research Group MITI, Klinikum rechts der Isar, Technische

Universität München, Munich, Germany

A. Schneider

e-mail: schneider@chir.med.tu-muenchen.de

D. Wilhelm · H. Feußner $(\square)$

Department of Surgery, Klinikum rechts der Isar,

Technische Universität München, Ismaningerstrasse 22,

Munich 81675, Germany

e-mail: feussner@chir.med.tu-muenchen.de work faster than the comparable standard dissector but produced significantly less mist.

Conclusion Ultrasonic shears are effective for bloodless tissue dissection but may impede surgical proceeding by mist production. The findings of this study demonstrate that emission of mist can be reduced not only by lowering the dissection power, resulting in a prolonged dissection time, but also by modifying the technical design of an instrument. Further development of ultrasonic cutting devices therefore should account for the desired results.

Keywords Minimally invasive surgery .

Mist production - Tissue dissection efficiency .

Ultrasound shears

Ultrasound dissection is an elegant, fast, and safe method of tissue dissection that has facilitated minimally invasive surgery [7]. Especially during large resections, a significant reduction in operating time with ultrasound dissection compared with electrosurgery devices can be achieved [1]. Moreover, ultrasonic dissection avoids the risks of monopolar electrocautery-like thermal injuries [2, 5].

However, ultrasound dissection also has its disadvantages: Cavitation leads to the production of mist. This is more acceptable than smoke because it vanishes much more rapidly than the smoke produced by high-frequency cautery. Nevertheless, the mist remains a disturbing side effect that may significantly impair visualization. Some concern also exists about the dissemination of vital (tumor) cells in the mist, as seen in smoke from electrocautery during laparoscopic interventions [3].

Currently, a new generation of ultrasound generators is available that purport to reduce the problem of mist 
production. The objective of this study was to analyze whether real differences in mist formation exist among the various ultrasonic dissectors offered on the market. Additionally, the efficiency of these shears was compared because mist formation certainly is dependent on the power applied.

Other studies already have assessed the dissection time required for laparoscopic bowel resection in an animal series, but in vivo assessments are difficult to standardize [1]. Further studies have analyzed the power of ultrasound dissectors to seal large arteries [4]. This study aimed to compare dissection efficiency and mist formation under strictly standardized in vitro conditions.

\section{Materials and methods}

\section{Equipment}

Four different laparoscopic ultrasound dissectors from three different producers were compared. Instrument $\mathrm{D}$ is an improved version of instrument $C$ particularly designed to produce less mist (Table 1).

The following features were adjusted to make the devices as comparable as possible. The power setting was adjusted to maximum on all the devices. The disposable instruments (A and B) were changed between the assessment for mist production and the assessment for efficiency. The reusable devices were cleaned and prepared according to the clinical standard. The specific features of the devices are listed in Table 1.

A comparison of all four instruments at maximum power was possible due to electromechanical characteristics: The power of an ultrasonic device depends not on its frequency or amplitude alone, but also on a combination of both. Indeed, coagulation, resulting from the cavitation effect, depends on the velocity of sound, which is defined by following formula:

Vnbsp $;=n b s p ; A_{\max } \times 2 \pi F \times \cos (2 \pi F t)$

where $V$ is the velocity of sound, $A$ is the amplitude, $F$ is the frequency, and $t$ is the time.

Test setup for quantifying mist production

A dedicated test setup was developed to assess mist production. In a hermetically sealed box, a small channel with a diameter of $6 \mathrm{~mm}$ for the dissection instrument was attached. Gas tightness was ensured with a rubber sealing. In the test box, a light-emitting diode (LED) and a photo transistor were attached above the dissection area with a specific circuit (Fig. 1) to assess the reduction of the light on the photo transistor by the mist (Fig. 2).

To reduce side effects in the light-measuring section due to visible light, components were chosen to measure above the visible light spectrum in the infrared wave length at $880 \mathrm{~nm}$. For this purpose a SFH485 LED (Osram Opto Semiconductors, Regensburg, Germany) and a photo transistor (OP505A, TTelectronics, Carrollton, TX, USA) on the opposite side were used, which work in even in this wavelength.

A layer of pure lard with dimensions (thickness, $1.5 \mathrm{~mm}$; width, $5 \mathrm{~mm}$ ) for dissection in one cut was positioned on a bench in the gastight plastic box. The shear was inserted into the opening, and the dissection was activated until the lard was completely cut. Infrared light transmission was assessed during the procedure and for another $15 \mathrm{~s}$ after its completion. The mist formation was evaluated as the percentage of reduction in infrared transmission.

Before each test, the baseline was precisely adjusted. For each instrument, a series of 10 measurements were

Table 1 Device specific parameters

\begin{tabular}{|c|c|c|c|c|}
\hline & \multicolumn{4}{|l|}{ Instruments } \\
\hline & A & $\mathrm{B}$ & $\mathrm{C}$ & $\mathrm{D}$ \\
\hline \multicolumn{5}{|l|}{ Working shaft } \\
\hline Diameter (mm) & 5.5 & 5.5 & 5.5 & 5.5 \\
\hline Length (mm) & 290 & 355 & 345 & 345 \\
\hline \multicolumn{5}{|l|}{ Active blade } \\
\hline Length (mm) & 9 & 9 & 13.5 & 13.5 \\
\hline Type & Blunt & Blunt, curved & Blunt, curved & Blunt, curved \\
\hline Approximate opening angle & $40^{\circ}$ & $50^{\circ}$ & $45^{\circ}$ & $45^{\circ}$ \\
\hline Use & Disposable & Disposable & Reusable & Reusable \\
\hline \multicolumn{5}{|l|}{ Special features } \\
\hline & & & HF connection & HF connection \\
\hline
\end{tabular}

$H F$ high frequency 


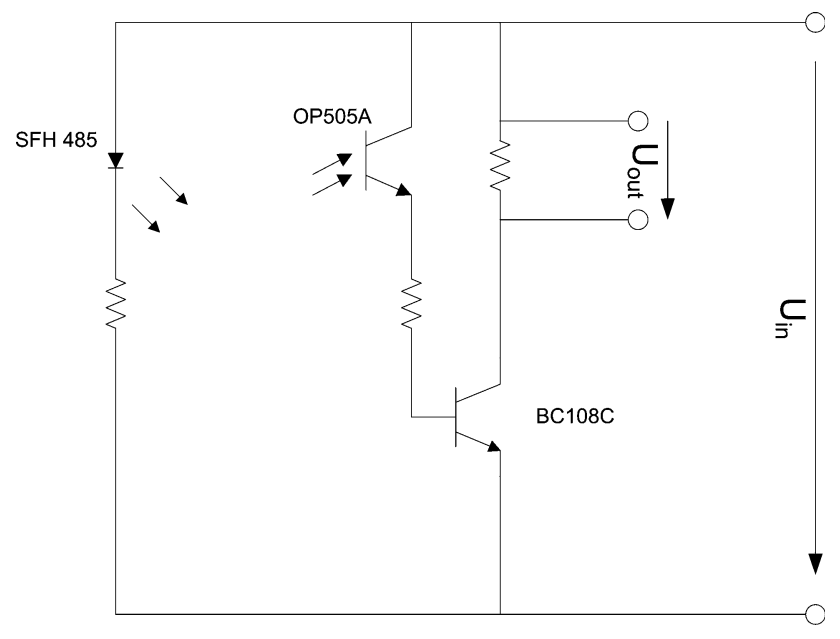

Fig. 1 Circuit for measurement of light attenuation. On the left side, the light-emitting diode is powered. The central part is the light analysis part with signal amplification. Uin is the supply voltage, and Uout is the measured signal

performed. Both the test box and the instruments were spring cleaned after each measurement.

\section{Evaluation of tissue dissection efficiency}

To evaluate the efficiency of the different dissection instruments, a steak of beef exactly $30 \mathrm{~mm}$ wide and $0.5 \mathrm{~cm}$ thick was cut completely at a right angle to the fibers with each of the shears. The parameters assessed during this analysis were the time needed for dissection (without time for readjustment of the dissection instrument) and the numbers of individual cuttings.
Mist production regarding the tissue

To assess the mist production during the dissection of different tissues, a steak of beef and pure lard of the same thickness $(0.5 \mathrm{~mm})$ and a width of $5 \mathrm{~mm}$ were dissected with the Harmonic Ace in the gastight test set using by same procedure used to evaluate mist production.

Statistical analysis

The nonparametric Kruskall-Wallis test was used to compare the light extinction of the four instruments in both groups (immediately after dissection, then $15 \mathrm{~s}$ after dissection) and to compare the relation of all the instruments with dissection time.

The Mann-Whitney $U$ test was used to analyze statistical significance for comparing the mist production of beef and lard. Statistical analysis was performed using SPSS 16.0 for Windows (SPSS Inc., Chicago, IL, USA), and $p$ values less than 0.05 were considered statistically significant.

\section{Results}

\section{Mist production}

Ultrasonic dissection of the lard layer required a mean time of $5.9 \mathrm{~s}$ with instrument A, $8.7 \mathrm{~s}$ with instrument B, $6.8 \mathrm{~s}$ with the newly developed instrument $\mathrm{D}$, and $7.18 \mathrm{~s}$ with the older version (instrument $\mathrm{C}$ ). The mist formation increased steadily until the end of the dissection process.
Fig. 2 Schematic drawing and test box for assessing mist production. Internally, the photo transistor is attached on the left upper side and the light-emitting diode (LED) on the opposite side. For cleaning aspects, the upper part and the dissection part are dividable
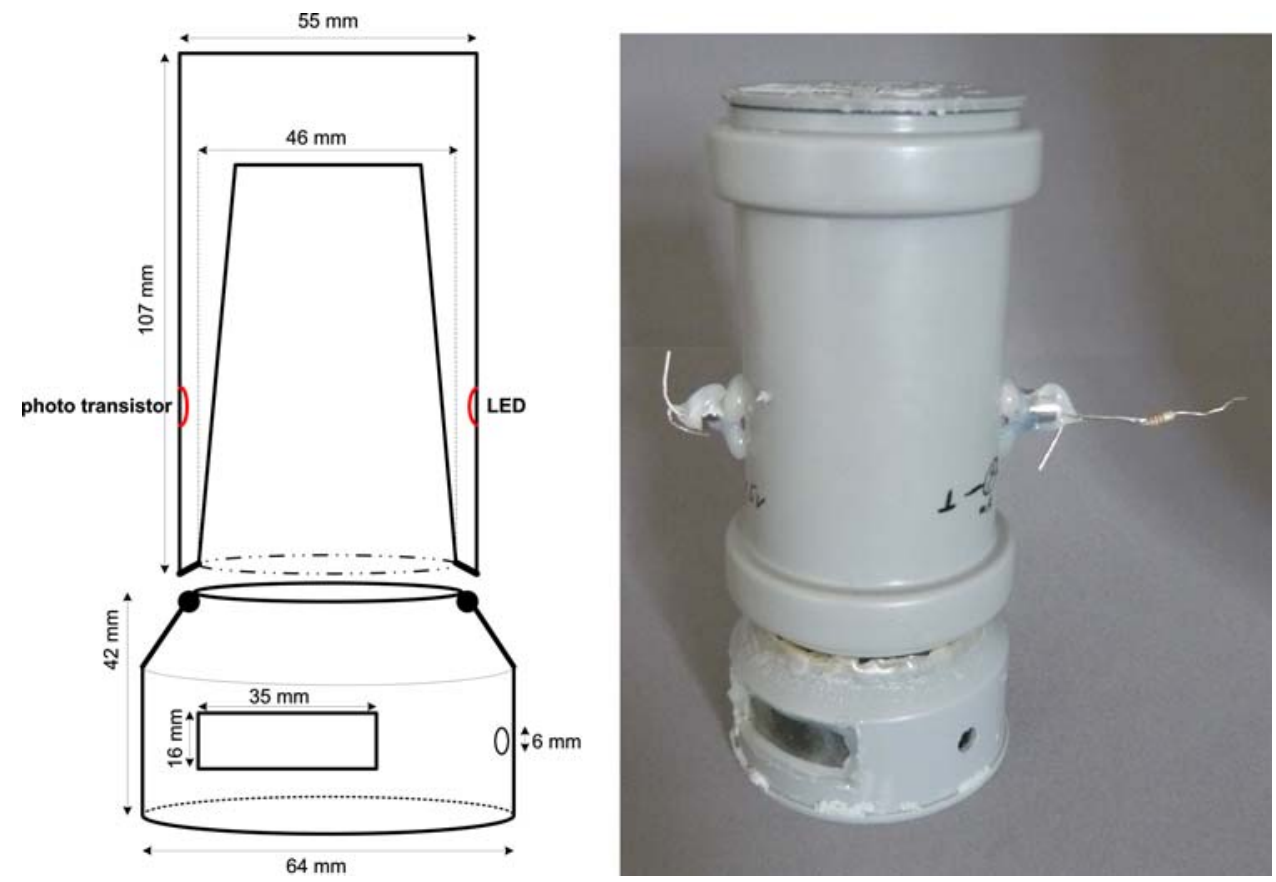


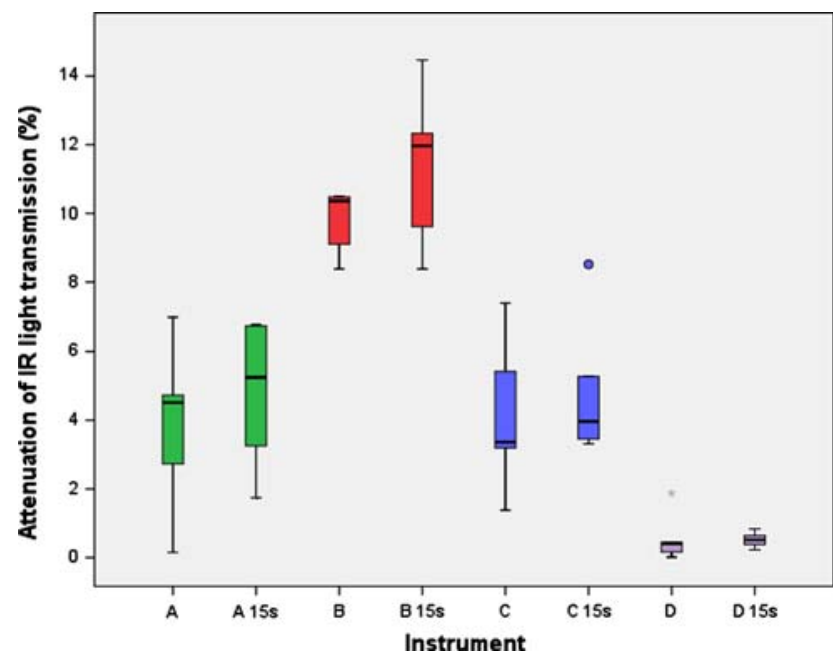

Fig. 3 Light attenuation during dissection of lard. For each instrument, the percentage of attenuation both immediately after cutting and $15 \mathrm{~s}$ later without instrument activity was recorded $(p<0.001)$

The reduction of light transmission was even slightly longer than active dissection. Accordingly, extinction was compared immediately after the coagulation, then after $15 \mathrm{~s}$ without instrument activity for recording mist spread.

As expected, the newly developed instrument $\mathrm{D}$ produced less mist with a mean attenuation of $0.56 \% \pm 0.6 \%$ immediately after dissection and $0.54 \% \pm 0.2 \%$ after another $15 \mathrm{~s}$. In terms of mist production, the conventional instrument $\mathrm{C}$ and instrument A were comparable $(3.58 \% \pm$ $2.1 \%$ vs. $3.82 \% \pm 2.6 \%$ immediately after dissection and $3.45 \% \pm 2.2 \%$ vs. $4.74 \% \pm 2.2 \% 15 \mathrm{~s}$ after dissection). Instrument B produced the most mist, with $11.23 \% \pm 3.7 \%$ in the immediate measurement and $13.73 \% \pm 6.2 \%$ in the delayed measurement (Fig. 3) $(p<0.001)$.

\section{Evaluation of efficiency}

To evaluate the efficiency of the shears, a steak of beef $30 \mathrm{~mm}$ wide and $5 \mathrm{~mm}$ thick was cut completely with each of the shears. Up to seven individual cuts per steak were needed for a complete cut, which depended on the length of each individual cut. The individual cut length was defined by the opening angle of the shears as well as by the length of the active surface.

The fastest device was instrument B (mean dissection time, of $8.5 \pm 1.4 \mathrm{~s}$ ), and the slowest was instrument A (mean dissection time, $18.5 \pm 2.7 \mathrm{~s}$ ). The improved instrument $\mathrm{D}$ was faster than the standard instrument $\mathrm{C}$ (mean dissection time, $16.0 \pm 1.5 \mathrm{~s}$ vs. $17.3 \pm 1.3 \mathrm{~s}$ ) (Fig. 4) $(p=0.001)$.

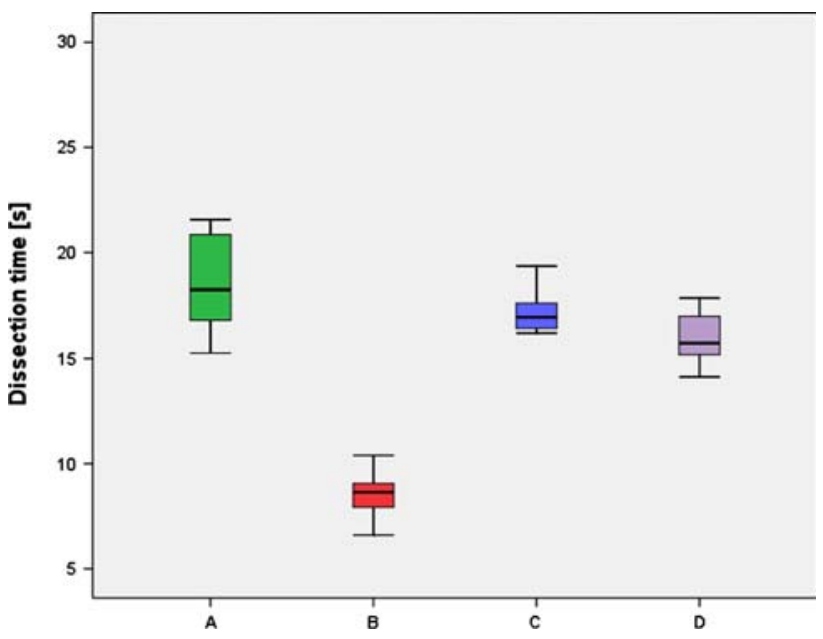

Fig. 4 Comparison of the different ultrasonic dissectors in terms of speed in cutting a steak of beef $0.5 \mathrm{~mm}$ wide and $30 \mathrm{~mm}$ long $(p=0.001)$

Mist production regarding the tissue

As expected, the lard produced significantly more mist than the strip of beef, showing a percentage reduction in light transmission of $11.2 \% \pm 3.7 \%$ vs. $2.99 \% \pm 1.8 \%$ $(p=0.002)$ immediately after dissection and $13.7 \% \pm$ $6.2 \%$ vs. $6.3 \% \pm 4.0 \%$ in the 15 -s delayed measurement $(p=0.026)$.

\section{Discussion}

Ultrasound dissection significantly enhanced the development of minimally invasive surgery because it permitted relatively quick tissue dissection without any blood loss. Currently, several devices on the market already have been compared in terms of their coagulation capacity and temperature safety profile $[1,6]$. However, a peculiarity of ultrasound dissection-mist formation-which sometimes is very disturbing during clinical application, to date has not been evaluated to the best of our knowledge. In some cases, especially with obese patients, it severely impedes visualization, and the pneumoperitoneum must be refilled repeatedly to regain a clear view of the operative site. In addition to reducing safety, this significantly prolongs the operation and eliminates, to a degree, the time-saving effect of ultrasound dissection.

An ideal ultrasound device should provide maximum coagulation speed with minimal mist formation. Neither aim can be reached completely because mist formation, en principe, is unavoidable. However, the question is whether an optimal compromise can be found. 
Quantification of mist formation is difficult. Evaluation in a clinical setting (e.g., animal experiments) is highly subjective. We, therefore, developed an in vitro test system based on light attenuation. Very low deviation occurs under identical test conditions, so our system seems to be suitable for comparing mist formation between different devices, although biologic material (lard and beef) was used.

Because mist formation correlates with the "cutting power" of the ultrasound dissector, we additionally determined the dissection velocity using a standardized strip of beef. It could be argued that this may be biased by biologic differences between the individual specimens of beef. This could perhaps play a major role if different anatomic regions are taken or if the beef of different animals is used. However, in our trial, only specimens of the same charges were taken. Accordingly, the test conditions should have been more or less identical.

The results confirmed the strong interdependence between dissection power and mist formation. Devices A and $\mathrm{C}$ were slower than device $\mathrm{B}$, but mist formation was markedly weaker as well. Inversely, device B was fast but led to a maximum of mist formation.

Most surprisingly, however, device D was not significantly slower than A or C, but its mist formation was clearly weaker. Evidently, potential still exists for designing new devices with a better quotient between velocity and mist formation, and manufacturers should be encouraged to optimize these systems further. For example, devices with a torsional ultrasonic vibration might be considered. However, we are not able to comment on the impact that different patterns of vibration (longitudinal or torsional) had on mist production in this study because torsionally activated instruments were not evaluated. We recognize that mist production strongly depends on the shape of the probe's distal part rather than on patterns of vibration. The shape that can reduce cavitation realizes reduction of mist.

As a practical recommendation for the surgeon, it should be mentioned that a fast instrument could be useful in large cavities. In a smaller space (e.g., in transanal endoscopic microsurgery), a fog-reduced device such as instrument $\mathrm{D}$ is preferable.

\section{References}

1. Bergamaschi R, Yavuz Y, Marvik R (2003) Laparoscopic bowel resection: a comparison of three ultrasonically activated devices. JSLS 7:19-22

2. Birch DW, Park A, Shuhaibar H (1999) Acute thermal injury to the canine jejunal free flap: electrocautery versus ultrasonic dissection. Am Surg 65:334-337

3. Champault G, Taffinder N, Ziol M, Riskalla H, Catheline JM (1997) Cells are present in the smoke created during laparoscopic surgery. Br J Surg 84:993-995

4. Clements RH, Palepu R (2007) In vivo comparison of the coagulation capability of SonoSurg and Harmonic Ace on 4-mm and 5-mm arteries. Surg Endosc 21:2203-2206

5. Gossot D, Buess G, Cuschieri A, Leporte E, Lirici M, Marvik R, Meijer D, Melzer A, Schurr MO (1999) Ultrasonic dissection for endoscopic surgery. The E.A.E.S. Technology Group. Surg Endosc $13: 412-417$

6. Kim FJ, Chammas MF Jr, Gewehr E, Morihisa M, Caldas F, Hayacibara E, Baptistussi M, Meyer F, Martins AC (2008) Temperature safety profile of laparoscopic devices: Harmonic ACE (ACE), Ligasure V (LV), and plasma trisector (PT). Surg Endosc 22:1464-1469

7. Yamakawa T, Kitano S, Kimura T, Matsumoto S (2002) New integrated ultrasonic surgical system, Sonosurg. Dig Endosc 14: $163-166$ 